\title{
Multiple facets of membrane lipids and the diversity of their action mode with special emphasis on the central nervous system
}

\author{
Jean-Marc ALESSANDRI*, Philippe GUESNET
}

INRA, Nutrition and Food Safety, Neurobiology of Lipids, 78352 Jouy-en-Josas, France

\begin{abstract}
This issue of Reproduction Nutrition Development was scheduled along with 8 papers on the metabolism of polyunsaturated fatty acids (PUFAs) and their physiological roles within the central nervous system. Topics focused on PUFAs from the n-3 series, especially on docosahexaenoic acid (DHA) which is particularly abundant in the cell membranes of brain and retina. Reviews and original articles emphasize the impact of n-3 PUFAs on the physical properties of membranes, the neuroprotective mechanisms via $\mathrm{Ca}^{2+}$ signalling in astrocytes, the $\mathrm{n}-3$ status of infants suffering of Attention Deficit Hyperactivity Disorder (ADHD) and the mental development of neonates in relation with maternal feeding. The DHA metabolism in humans and its endogenous production from alpha-linolenic acid is reviewed. Besides, it is described and discussed how the generation of PUFAderived mediators is modulated by the retinoic acid-receptor signalling via activation of cognate phospholipase activities. The sensitivity and recovering of neurons to oxidative stress is also addressed in this volume, through the description of a model of iron-induced injury in the rat brain. The diversity of these 8 articles illustrates the multiple roles of PUFAs within the central nervous system.
\end{abstract}

arachidonic acid / astrocytes and calcium signalling / Attention Deficit Hyperactivity Disorder / central nervous sytem / docosahexaenoic acid / endogenous conversion / membrane physical properties / milk and infant mental development / omega-3 and omega-6 fatty acids / oxidative stress / phospholipases / raft / retinoic acid

\section{INTRODUCTION}

This part of volume of Reproduction Nutrition Development is devoted to several facets of the functional roles of fatty acids and to their metabolism within the nervous system. The multi-disciplinarity of the topics covered within this issue reflects the extraordinary diversity of the regulation pathways in which fatty acids, especially the polyunsaturated fatty acids (PUFAs), are closely implicated, either directly as membrane components or indirectly as precursors of active metabolites. Probably, there is no other example of nutrients that cumulate the particular features of being indispensable components of membrane genesis, modulators of the membrane dynamics and transmembrane functions, and being at the same time endogenous mediators in

\footnotetext{
* Corresponding author: Jean-Marc.Alessandri@jouy.inra.fr
} 
cell signalling and gene expression. These multiple features of PUFAs - which are not mutually exclusive - underlie the adequate perinatal development of the CNS, contribute to protect the brain from pathological stimuli, and finally participate in the behavioral functions throughout the lifespan $[1,2]$.

Starting with the molecular scale, the article by Stillwell et al. [3] shows that docosahexaenoic acid (DHA, 22:6n-3), the longest and most unsaturated fatty acid found in membranes, is incorporated into the different phospholipid molecular species according to a tissue-specific distribution. It is consensually recognised that the concentration of DHA affects the physical properties of membranes, but generations of researchers have debated, and still debate, on how. Stillwell et al. offer an overview of the means by which DHA modulates the membrane architecture and physical properties, through the complementary and evolutive notions of membrane curvature, fluidity, deformability, microdomain organisation and rafts. In particular, it is explained in this article how the interactions between DHA and cholesterol, which produce opposite effects on the lateral partitioning of ordered and disordered domains, may affect the membrane structure and hence the properties of the embedded proteins. From this view, the most thoroughly studied of the integral membrane receptors by far has been rhodopsin. The concept has emerged that the photo-induced conformational changes of rhodopsin are primarily enhanced by DHA-rich phospholipids and secondarily slowed down by cholesterol [4]. This model probably provides the most comprehensive molecular basis of the mechanism by which the DHA status may be related to the development of the visual function during infancy.

One important challenge in human nutrition is the question of whether the brain obtains its DHA directly from dietary fats, body stores, or endogenous conversion of the $n-3$ dietary precursor, $\alpha$-linolenic acid $(18: 3 n-3)$. The question of the conversion pathway is addressed by Burdge and Calder [5] who reviewed recent studies that have been conducted in several countries. Adult men and women volunteers had received tracer doses of a stable isotope of $\alpha$-LNA, enabling the investigators to examine the short-term metabolic fate of $\alpha$-LNA, that is either storage, oxidation or conversion into the major long-chain derivatives, eicosapentaenoic acid (EPA, 20:5n-3) and DHA. Both the short-term studies using stable isotopes and the more traditional long-term studies of dietary intakes indicate that $\alpha$-LNA is a limited source of long-chain derivatives in adults, especially with regards to the production of DHA and its incorporation into the bloodstream lipids. More surprisingly, the fractional conversion of $\alpha$-LNA appears to be greater in women than in men, raising the unresolved question of the possible role of sex steroids in the regulation of PUFA metabolism. In any event, one important conclusion brought about by the review of Burdge and Calder is that adequate intakes of preformed DHA could be more efficient than intakes of $\alpha$-LNA for maintaining optimal tissue functions throughout adult life.

The impact of long-chain PUFAs on health, especially on mental health, increasingly appears of capital importance. Altered levels of PUFAs in blood lipids have been reported in a variety of neuropathologies, and several epidemiological studies have shown that frequent consumption of n-3 fatty acids may be inversely linked to neuropsychiatric and neurodegenerative diseases [6, 7]. Although the causes of the observed abnormalities of fatty acids in sufferers of neuropathologies are mostly unknown, it is considered that normalisation of the PUFA status through dietary supplementation could be beneficial to prevent these diseases or could help improve the cognitive and behavioral functions. This nutritional approach is proposed by Young et al. [8] who specifically addressed in their article the Attention Deficit Hyperactivity Disorder (ADHD), a neuropsychiatric disease that is commonly associated 
with decreased blood n-3 fatty acid levels. In particular, ADHD patients present a higher ratio of arachidonic acid (AA, 20:4n-6) to EPA than normal. The objective of Young et al. was to raise the levels of the longchain n-3 polyunsaturated fatty acids in the serum phospholipids of adults with diagnosed ADHD, and thereby to decrease the AA/EPA ratio. As discussed in connection with the review by Burdge and Calder [5], Young et al. examined whether intakes of flax oil rich in $\alpha$-LNA can be as much efficient to raise the $n-3$ status of ADHD patients as using fish oil rich in DHA and EPA. Their data indicate that treatment with fish oil increases the EPA and DHA levels and decreases the AA/EPA ratio [8]. The effect of flax oil on EPA and DHA being of very low extent, the actual metabolic ability of ADHD patients to convert $\alpha$-LNA into its longer-chain derivatives should be questioned. Clearly, this original study shows that if the n-3 PUFA status needs to be optimised in ADHD patients, fish oil will be much more efficient than vegetable oil.

The PUFA status also results from the whole energy and lipid metabolism. Since dietary fats are generally composed of blends of saturated, monounsaturated and polyunsaturated fatty acids, Rioux et al. [9] examined the impact of dietary myristic acid (14:0) on the PUFA levels within rat tissues. They show that physiological intakes of 14:0 produce an increase of the PUFA contents. PUFAs from both the n- 6 and $n-3$ series increased, but this effect only affected fatty acids made of 20 carbons [9]. Hence, dietary myristic acid may participate in the regulation of PUFA metabolism, especially those serving as potential precursors of bioactive metabolites (eicosanoids). This nutritional aspect is important since, once incorporated into neuronal membrane phospholipids, PUFAs are susceptible to be released under the action of hormonal stimuli and to generate an array of mediators that may trigger a cascade of events [10]. Thereby, the enzymatic release of PUFAs from membranes constitutes a key process in cell signalling, that has an impact on cell physiology, growth, differentiation, or apoptosis. Lipid signalling is reviewed or addressed in three studies within the present volume.

The commentary article by Farooqui and Horrocks [11] focuses on the role of phospholipases in the release of lipid mediators which are implicated in the differentiation commitment of neuronal cells. The authors have particularly studied the coupling between the activation of phospholipases and the retinoic acid receptor-mediated gene transcription. The retinoic acid receptor pathway is known to activate genes involved in lipid metabolism [12] and to stimulate morphological differentiation of neuronal cells [13]. Their striking conclusion is that retinoic acid increases the transcription and thus the activity of nuclear phospholipases, resulting in the hydrolysis of nuclear phospholipids without an effect on non-nuclear membranes. Farooqui and Horrocks suggest that the nucleus controls through the phospholipase pathway the redistribution of AA from nuclear phospholipids into other cellular membranes. Thus, nuclear membrane phospholipids are substrates of phospholipases specifically operating within the nucleus for the release of AA and diacylglycerol (DAG) and for the generation of AA-derived mediators. These metabolites are normally involved in cell proliferation and differentiation. However in pathological conditions of overproduction, they may trigger inflammatory, oxidative and neurodegenerative processes.

The release of PUFAs from astrocyte membranes is induced by different phospholipases, most of the AA release being stimulated by $\mathrm{Ca}^{2+}$ while that of DHA is $\mathrm{Ca}^{2+}$-independent [14]. Therefore, the release of $\mathrm{Ca}^{2+}$ from its internal stores plays a crucial role in the specific activation of phospholipases, leading to the release of PUFAs and to their dispatching towards specific signalling pathways. Furthermore, it increasingly appears that oscillations of $\mathrm{Ca}^{2+}$ concentrations are crucial for the communication between glial cells and neurons and for 
synchronising the physiological responses within the brain [15]. In return, do the PUFAs have an influence on the $\mathrm{Ca}^{2+}$ signalling in astrocytes? Sergeeva et al. [16] review and address this fascinating question by measuring the agonist-induced $\mathrm{Ca}^{2+}$ signals (influx, release and store replenishment) in astrocytes beforehand incubated with AA, EPA or DHA. The very comprehensive model of the authors reveals that PUFAs modulate the steady state level of internal $\mathrm{Ca}^{2+}$ and hence the ability of astrocytes to respond to further activation by external stimuli. The conclusion of this study is that the "insensibilisation" brought about by free PUFAs may protect the surrounding cells from pathological overstimulation, offering a relevant and remarkable explanation of the neuroprotective mechanisms of AA and DHA.

However, PUFAs also present a dark side, including their high sensitivity to oxidative reactions which may be generated in neuropathological conditions, and which may produce adverse effects in the brain. Models of oxidative injury are needed to identify the cellular targets of reactive oxygen species (ROS) within brain regions. Since chronic iron accumulation is thought to be associated with oxidative stress in several neurodegenerative diseases, Ong et al. [17] studied the generation of toxic aldehyde compounds that are produced from the release and peroxidation of PUFAs in response to intracerebroventricular injection of ferrous ammonium citrate. They observed by microscopy the iron-induced immunolabelling of hydroxynonenal (HNE) and of cytosolic phospholipase $\mathrm{A}_{2}\left(\mathrm{cPLA}_{2}\right)$ in the pyramidal neurons of the rat hippocampus. HNE positive cells exhibited features of injured neurons, showing that this model mimics situations where there is increased brain oxidative stress due to iron accumulation in vivo. Furthermore, the data give information on the transient induction of $\mathrm{cPLA}_{2}$ and on the anti-peroxidative mechanisms that are able to clear the HNE immunoreactivity within weeks postinjection. One interesting conclusion of
Ong et al. is that rat neurons have a remarkable ability to tolerate and recover from a single episode of severe oxidative stress.

The impact of dietary PUFAs, especially of AA and DHA, on the neurodevelopmental outcomes in neonates receiving breast milk or milk replacers is still a controversial question. Although breast milk feeding is considered as being the "golden standard", human milk is submitted to high variability regarding the concentrations of $\mathrm{AA}$ and DHA among populations and among individuals from the same population [18]. Feeding with milk replacers however raises the question of supplementing with DHA alone, or with AA and DHA, and if so in which ratio. In any case, the effects, beneficial or not, on the development of mental and visual functions need to be evaluated. The study by Lauritzen et al. [19] examined if an increased content of DHA in the milk of Danish mothers with a habitual fish intake below the Denmark population median and receiving a fish oil-supplementation, may affect the mental development of the infants as compared to a control group receiving olive oil-supplementation and to a reference group with median fish intake. In particular, the authors evaluated the outcomes on problem solving at nine months of age and early language acquisition. The data indicate that fish oil-supplementation of the lactating mothers may have a slightly positive effect on the problem solving ability of the infants, but only in girls. However, maternal fish oil-supplementation also resulted in a transient reduction in vocabulary comprehension at one year of age, especially in boys, and word production at one year was inversely associated with DHA levels in infant erythrocytes. Therefore, the study by Lauritzen et al. shows that increasing the DHA-content of breast-milk may affect the early language acquisition of breast-fed infants. Although DHA-related long-term adverse effects have not been observed until now, the observations of Lauritzen et al. highlight that it is necessary to optimise the dietary supplies in PUFAs of lactating mothers, 
especially with regards to the relative concentrations of long-chain PUFAs from both the n-6 and n-3 series.

\section{REFERENCES}

[1] Lauritzen L, Hansen HS, Jùrgensen MH, Michaelsen KF. The essentiality of long chain n-3 fatty acids in relation to development and function of the brain and retina. Prog Lipid Res 2001, 40: 1-94.

[2] Alessandri JM, Guesnet P, Vancassel V, Astorg P, Denis I, Langelier B, Aïd S, PoumèsBallihaut C, Champeil-Potokar G, Lavialle M. Polyunsaturated fatty acids in the nervous system: evolution of concepts and nutritional implications throughout life. Reprod Nutr Dev 2004, 44: 509-538.

[3] Stillwell W, Shaikh SR, Zerouga M, Siddiqui R, Wassall SR. Docosahexaenoic acid affects cell signaling by altering lipid rafts. Reprod Nutr Dev 2005, 45: 559-579.

[4] Niu SL, Mitchell DC, Litman BJ. Manipulation of cholesterol levels in rod disk membranes by methyl-beta-cyclodextrin: effects on receptor activation. J Biol Chem 2002, 277 : 20139-20145.

[5] Burdge GC, Calder PC. Conversion of alphalinolenic acid to longer-chain polyunsaturated fatty acids in human adults. Reprod Nutr Dev 2005, 45: 581-597.

[6] Horrocks LA, Farooqui AA. Docosahexaenoic acid in the diet: its importance in maintenance and restoration of neural membrane function. Prostaglandins Leukot Essent Fat Acids 2004, 70: 361-372.

[7] Young G, Conquer J. Omega-3 fatty acids and neuropsychiatric disorders. Reprod Nutr Dev 2005, 45: 1-28.

[8] Young GS, Conquer JA, Thomas R. Effect of randomized supplementation with high dose olive, flax or fish oil on serum phospholipid fatty acid levels in adults with attention deficit hyperactivity disorder. Reprod Nutr Dev 2005, 45: 549-558.

[9] Rioux V, Catheline D, Bouriel M, Legrand P. Dietary myristic acid at physiologically relevant levels increases the tissue content of
C20:5 n-3 and C20:3 n-6 in the rat. Reprod Nutr Dev 2005, 45: 599-612.

[10] SanGiovanni JP, Chew EY. The role of omega-3 long-chain polyunsaturated fatty acids in health and disease of the retina. Prog Retinal Eye Res 2004, 24: 87-138.

[11] Farooqui AA, Horrocks LA. Signaling and interplay mediated by phospholipases A2, C, and D in LA-N-1 cell nuclei. Reprod Nutr Dev 2005, 45: 613-631.

[12] Anderson SP, Dunn C, Laughter A, Yoon L, Swanson C, Stulnig TM, Steffensen KR, Chandraratna RAS, Gustafsson JA, Corton JC. Overlapping transcriptional programs regulated by the nuclear receptors peroxisome proliferator-activated receptor alpha, retinoid $\mathrm{X}$ receptor, and liver $\mathrm{X}$ receptor in mouse liver. Mol Pharmacol 2004, 66: 1440-1452.

[13] Malik MA, Blusztajn JK, Greenwood CE. Nutrients as trophic factors in neurons and the central nervous system: Role of retinoic acid. J Nutr Biochem 2000, 11: 2-13.

[14] Strokin M, Sergeeva M, Reiser G. Docosahexaenoic acid and arachidonic acid release in rat brain astrocytes is mediated by two separate isoforms of phospholipase $\mathrm{A} 2$ and is differently regulated by cyclic AMP and $\mathrm{Ca}^{2+}$. Br J Pharmacol 2003, 139: 1014-1022.

[15] Carmignoto G. Reciprocal communication systems between astrocytes and neurones. Prog Neurobiol 2000, 62: 561-581.

[16] Sergeeva M, Strokin M, Reiser G. Regulation of intracellular calcium levels by polyunsaturated fatty acids, arachidonic acid and docosahexaenoic acid, in astrocytes involving different types of phospholipase A2. Reprod Nutr Dev 2005, 45: 633-646.

[17] Ong WY, Ling SF, Yeo JF, Chiueh CC, Farooqui AA. Injury and recovery of neurons in the rat hippocampus after a single severe oxidative stress induced by intracerebroventricular ferrous ammonium citrate injection. Reprod Nutr Dev 2005, 45: 647-662.

[18] Innis SM. Human milk and formula fatty acids. J Pediatr 1992, 120: S56-S61.

[19] Lauritzen L, Jørgensen MH, Olsen SF, Straarup EM, Michaelsen KF. Maternal fish oil supplementation in lactation: Effect on developmental outcome in breast-fed infants. Reprod Nutr Dev 2005, 45: 535-547. 